David Arcoya · José Carmona

\title{
On two problems studied by A. Ambrosetti
}

\author{
Dedicated to Antonio Ambrosetti on the occasion of his sixtieth birthday
}

Received September 14, 2005 and in revised form December 11, 2005

\begin{abstract}
We study the Ambrosetti-Prodi and Ambrosetti-Rabinowitz problems. We prove for the first one the existence of a continuum of solutions with shape of a reflected C ( $\supset$-shape). Next, we show that there is a relationship between these two problems.
\end{abstract}

Keywords. Ambrosetti-Prodi and Ambrosetti-Rabinowitz problems, topological degree, a priori bound

\section{Introduction}

In the early seventies, Antonio Ambrosetti wrote in collaboration with Giovanni Prodi [3] and Paul H. Rabinowitz [4] two of the most seminal papers in the theory of nonlinear functional analysis. Both of them have become key-stones in the study of the existence and multiplicity of solutions for nonlinear P.D.E.

The problem considered in [3] is

$$
\begin{aligned}
-\Delta u & =f(u)+h, & & x \in \Omega, \\
u & =0, & & x \in \partial \Omega,
\end{aligned}
$$

where $f$ is, roughly speaking, a nonlinearity whose derivative crosses the first eigenvalue associated with the Laplacian operator with zero Dirichlet boundary condition. By using their abstract result, they are able to describe the exact number of solutions in terms of $h$.

On the other hand, in [4], using the Mountain Pass Theorem, the authors prove the existence of a positive (nontrivial and nonnegative) solution for the problem

$$
\begin{aligned}
-\Delta u & =f(x, u), & & x \in \Omega, \\
u & =0, & & x \in \partial \Omega,
\end{aligned}
$$

where, roughly speaking, $f$ is a superlinear nonlinearity $\left(\lim _{u \rightarrow+\infty} f(x, u) / u=+\infty\right)$ with $f(x, 0)=0$ for a.e. $x \in \Omega$ and with zero derivative with respect to $u$ at zero.

D. Arcoya: Departamento de Análisis Matemático, Universidad de Granada, 18071 Granada, Spain; e-mail: darcoya@ugr.es

J. Carmona: Departamento de Álgebra y Análisis Matemático, Universidad de Almeria, Almeria, Spain; e-mail: jcarmona@ual.es

Mathematics Subject Classification (2000): Primary 35J65; Secondary 35J25, 58E07 
These problems are known in the literature respectively as "Ambrosetti-Prodi" and "Ambrosetti-Rabinowitz" problems. Given the large amount of papers written on this subject, it is an almost impossible task to come up with an original view of them. Our wish here is to convince the reader that these "Ambrosetti problems" are not so different as they seem.

The ideas rely on the study of some quasilinear extensions of the results in [5] (see also [6]). In [5] we study the case of a nonvariational differential operator, while in [6] the $p$-laplacian operator $(p>1)$ is considered. The intrinsic difficulties of those quasilinear equations are the reason for developing a new bifurcation approach to the AmbrosettiProdi problem. Moreover, we deduce from it the quasilinear Ambrosetti-Rabinowitz result, unifying in this way those two classical results. As a tribute to the pioneering works by Ambrosetti et al. we devote this note to a survey of these results in the simpler semilinear case. We remark explicitly that, in this case, the interest is to give a new perspective to applications of the Leray-Schauder topological degree, in conjunction with the remarkable a priori bound of B. Gidas and J. Spruck [16], instead of variational methods. The interested reader can see [5] for the more complicated nonvariational quasilinear case.

\section{Continua of solutions for Ambrosetti-Prodi type problems}

In [3] (see also [2]), A. Ambrosetti and G. Prodi, using some results on the global inversion of differentiable mappings between Banach spaces, proved the following existence result for (1). In order to state it, we denote by $\left\{\lambda_{k}\right\}$ the sequence of eigenvalues for the Laplacian operator with zero Dirichlet boundary condition.

Theorem 2.1 (Ambrosetti-Prodi, 1972). Let $\Omega \subset \mathbb{R}^{N}$ be a bounded domain with $C^{2, \alpha}$ boundary $(0<\alpha<1)$ and let $f \in C^{2}(\mathbb{R})$ satisfy:

(i) $f(0)=0$,

(ii) $f^{\prime \prime}(s)>0$ for every $s \in \mathbb{R}$,

(iii) $\lim _{s \rightarrow-\infty} f^{\prime}(s)=f^{\prime}(-\infty)<\lambda_{1}<f^{\prime}(+\infty)=\lim _{s \rightarrow+\infty} f^{\prime}(s)<\lambda_{2}$.

Then there exists a connected and closed $C^{1}$-manifold, $\mathcal{M}$, of codimension 1 in $C^{0, \alpha}(\bar{\Omega})$ such that $C^{0, \alpha}(\bar{\Omega}) \backslash \mathcal{M}$ has exactly two connected components, $\mathcal{A}_{1}, \mathcal{A}_{2}$, with the following properties:

(a) if $h \in \mathcal{A}_{1}$, then problem (1) has no solution in $C^{2, \alpha}(\bar{\Omega})$,

(b) if $h \in \mathcal{A}_{2}$, it has exactly two solutions in $C^{2, \alpha}(\bar{\Omega})$,

(c) if $h \in \mathcal{M}$, it admits a unique solution in $C^{2, \alpha}(\bar{\Omega})$.

Thus, a precise description of the number of solutions of (1) is given. This result has been the motivation of a very large amount of work on the number of solutions of b.v.p. with nonlinearities $f(x, u)$ whose derivative jumps the first eigenvalue $\lambda_{1}$ or higher eigenvalues. It would be impossible to give here a complete list of references, but, at least, we wish to cite some of the most classical ones and we refer the reader to the surveys by D. G. de Figueiredo [11, 12] for a more extensive list. In [8], M. S. Berger and E. Podolak write the 
function $h$ as $h=t \varphi+\tilde{h}$, where $t \in \mathbb{R}, \varphi$ is a positive function and $\tilde{h}$ is orthogonal to $\varphi$ in $L^{2}(\Omega)$. Existence and multiplicity of solutions are described in terms of the values of $t$. Fixed point theory is applied by H. Amann and P. Hess in [1] and also by S. Fučik [14] who introduces the term of jumping nonlinearity. In particular, in [1], $f: \bar{\Omega} \times \mathbb{R} \rightarrow \mathbb{R}$ is a continuous function such that there exist positive functions $f_{ \pm \infty}^{\prime} \in L^{\infty}(\Omega)$ satisfying

$$
\lim _{s \rightarrow \pm \infty} \frac{f(x, s)}{s}=f_{ \pm \infty}^{\prime}(x) \quad \text { uniformly in } x \in \Omega .
$$

The result in [1] states:

Theorem 2.2. Suppose that $\varphi \in C^{0, \alpha}(\bar{\Omega})$ with $\varphi>0$ in $\Omega$. Assume also (3) and that there exists a positive constant $\varepsilon$ such that for a.e. $x \in \Omega$,

$$
f_{-\infty}^{\prime}(x)<\lambda_{1}-\varepsilon<\lambda_{1}+\varepsilon<f_{+\infty}^{\prime}(x)<\lambda_{2}-\varepsilon .
$$

Then there exists $t^{*} \in \mathbb{R}$ with the following properties:

(a) if $t>t^{*}$, the problem

$$
\begin{aligned}
-\Delta u & =f(x, u)+t \varphi, & & x \in \Omega, \\
u & =0, & & x \in \partial \Omega,
\end{aligned}
$$

has no solution,

(b) if $t=t^{*}$, it has at least one solution,

(c) if $t<t^{*}$, it admits at least two solutions.

A different approach based on Morse theory was given by H. Berestycki [7]. We must also mention the papers by D. G. de Figueiredo and S. Solimini [13] and K. C. Chang [10] where the case of subcritical superlinear nonlinearities jumping all eigenvalues $\lambda_{k}$ is considered, i.e. for some $\varepsilon>0$,

$$
f_{-\infty}^{\prime}(x)<\lambda_{1}-\varepsilon<f_{+\infty}^{\prime}(x) \equiv+\infty, \quad \text { a.e. } x \in \Omega .
$$

The main tool in [13] is the Mountain Pass Theorem, developed for the AmbrosettiRabinowitz problem. Indeed, they prove that one solution of $\left(P_{t}\right)$ can be obtained by the sub-super-solution method. As clarified by H. Brézis and L. Nirenberg [9], such a solution is a local minimum of the associated Euler functional with respect to the $H_{0}^{1}(\Omega)$ topology. Then the second one is obtained by application of the cited Mountain Pass Theorem (provided that, in addition, the nonlinearity $f$ satisfies the technical AmbrosettiRabinowitz condition [4] for the standard Palais-Smale condition). We also remark that the case of a critical superlinear nonlinearity is studied in [9] by using this variational technique.

The motivation of our results lies in the study of some quasilinear elliptic equations ([5, 6]). Other works on quasilinear Ambrosetti-Prodi problems are [17, 18]. The particular difficulties of these do not allow one to extend the previous ideas and, consequently, we have developed a new approach based on proving the existence of a continuum of solutions with $\supset$-shape (see Remark 2.77). We consider two classes of nonlinearities: those 
where the asymptotically linear nonlinearity $f$ satisfies (3) and (4), and the superlinear case of nonlinearities satisfying (3) and (5). In addition, in the latter case, we suppose that there exist $h \in L^{\infty}(\Omega)$ and $1<p<2^{*}-1$ such that

$$
\lim _{s \rightarrow+\infty} \frac{f(x, s)}{s^{p}}=h(x)>c>0 \quad \text { uniformly in } x \in \Omega .
$$

Here $2^{*}$ stands for the critical Sobolev exponent, i.e. $2^{*}=2 N /(N-2)$ if $N \geq 3$, while $2^{*}=+\infty$ for $N=2$.

The proof of our result applies the sub-super-solution method and the Leray-Schauder degree in conjunction with the following a priori bound on the $C^{0}(\bar{\Omega})$-norm of the solutions.

Lemma 2.3. Let $\varphi \in L^{\infty}(\Omega)$ be a positive function. Suppose that either

(i) conditions (3) and (4) hold, or

(ii) $f$ satisfies (3), (5) and (6).

Then the solutions of $\left(P_{t}\right)$ are uniformly bounded on compact sets of $t$, i.e., for every compact interval $\Gamma \subset \mathbb{R}$, there exists $c \in \mathbb{R}$ such that every solution u of $\left(P_{t}\right)$ with $t \in \Gamma$ satisfies

$$
|u(x)| \leq c, \quad \forall x \in \Omega .
$$

Remark 2.4. In the case of superlinear nonlinearities satisfying assumptions (ii), the above a priori bound is an easy extension of the result by B. Gidas and J. Spruck in [16].

Proof of Lemma 2.3. In the case of assumptions (i), the proof is standard [2]. We prove the lemma if (ii) holds. First we show that, given a compact real interval $\Gamma$, there exists a positive constant $c$ such that

$$
u(x)>-c, \quad x \in \Omega,
$$

for every solution $u$ of $\left(P_{t}\right)$ with $t \in \Gamma$. In order to prove this a priori bound, we observe that taking $u^{-} \equiv \min \{u, 0\}$ as test function in the equation satisfied by $u$, and by using hypothesis (5), we get a uniform bound of $u^{-}$in the $H_{0}^{1}(\Omega)$-norm. Consider now, for each $k \in \mathbb{R}$, the function $G_{k}$ given by

$$
G_{k}(s)= \begin{cases}s+k & \text { if } s \leq-k \\ 0 & \text { if }-k<s \leq k \\ s-k & \text { if } k<s\end{cases}
$$

Thus, taking $v=G_{k}\left(u^{-}\right)$as test function in the equation satisfied by $u$ we obtain

$$
\int_{\Omega}\left|\nabla G_{k}\left(u^{-}\right)\right|^{2}=\int_{\Omega_{k}}\left(f\left(x, u^{-}\right)+t \varphi\right) G_{k}\left(u^{-}\right),
$$

where $\Omega_{k} \equiv\{x \in \Omega: u(x)<-k\}$. From (5), there exists a positive constant $C$ such that

$$
f(x, s)+t \varphi \geq C s, \quad \forall s \leq-k, \forall t \in \Gamma .
$$

We deduce from the above that

$$
\int_{\Omega}\left|\nabla G_{k}\left(u^{-}\right)\right|^{2} \leq C \int_{\Omega_{k}}\left|u^{-}\right|\left|G_{k}\left(u^{-}\right)\right| .
$$


Using now the Sobolev and Hölder inequalities, we get for some new constant $C$ and $r>2 N /(N+2)$,

$$
\left\|G_{k}\left(u^{-}\right)\right\|_{2^{*}}^{2} \leq C\left\|u^{-}\right\|_{r}\left\|G_{k}\left(u^{-}\right)\right\|_{2^{*}}\left(\text { meas } \Omega_{k}\right)^{1-1 / r-1 / 2^{*}} .
$$

(For $r \geq 1$, we denote by $\|\cdot\|_{r}$ the usual norm of the Lebesgue space $L^{r}(\Omega)$.) Notice now that for every $l \geq k,\left|G_{k}\left(u^{-}\right)\right| \geq l-k$ in $\Omega_{l}$, which implies that

$$
(l-k)\left(\text { meas } \Omega_{l}\right)^{1 / 2^{*}} \leq C\left\|u^{-}\right\|_{r}\left(\text { meas } \Omega_{k}\right)^{1-1 / r-1 / 2^{*}},
$$

or equivalently that

$$
\text { meas } \Omega_{l} \leq \frac{C\left\|u^{-}\right\|_{r}^{2^{*}}\left(\text { meas } \Omega_{k}\right)^{2^{*}-1-2^{*} / r}}{(l-k)^{2^{*}}} .
$$

Using ideas of Stampacchia [19], we deduce the existence of a positive constant $c$ such that $\left\|u^{-}\right\|_{\infty} \leq c$ for every solution $u$ of $\left(P_{t}\right)$ with $t \in \Gamma$. Thus, (7) has been proved.

On the other hand, define $v=u+c \geq 0$ and $\tilde{f}(x, s)=f(x, s-c)$. Then $v$ satisfies

$$
\begin{aligned}
-\Delta v & =\tilde{f}(x, v)+t \varphi, & & x \in \Omega, \\
v & =c, & & x \in \partial \Omega .
\end{aligned}
$$

We observe that the result in [16] remains true for solutions of the equation with bounded Dirichlet data instead of zero Dirichlet data. This gives the existence of $\tilde{c} \in \mathbb{R}^{+}$such that $v(x) \leq \tilde{c}$ for every $x \in \Omega$, i.e. $u$ is bounded from above.

We also need the following abstract theorem about the existence of a つ-shaped continuum. The proof can be found in [5].

Lemma 2.5. Let $E$ be a Banach space and $T: \mathbb{R} \times E \rightarrow E$ a compact operator. Denote by $\Sigma$ the set of pairs $(t, u) \in \mathbb{R} \times E$ such that $u$ is a solution of

$$
u-T(t, u)=0 .
$$

Let $U$ be a bounded subset in E such that (8) has no solution on $\partial U$ while $t \in[a, b]$. Assume also that, for $t=b$, (8) has no solution in $\bar{U}$. Let $U_{1} \subset U$ be such that, for $t=a$, (8) has no solution on $\partial U_{1}$ and $\operatorname{deg}\left(I-T(a, \cdot), U_{1}, 0\right) \neq 0$. Then there exists a continuum $C$ in $\Sigma$ such that

$$
C \cap\left(\{a\} \times U_{1}\right) \neq \varnothing, \quad C \cap\left(\{a\} \times\left(U \backslash \overline{U_{1}}\right)\right) \neq \emptyset .
$$

Now, we prove the main result of this section.

Theorem 2.6. Let $\varphi \in L^{\infty}(\Omega)$ be a positive function and let $f: \bar{\Omega} \times \mathbb{R} \rightarrow \mathbb{R}$ be a $C^{1}$-function satisfying either

(i) conditions (3) and (4), or

(ii) conditions (3), (5) and (6). 
Then $t^{*} \equiv \sup \left\{t \in \mathbb{R}:\left(P_{t}\right)\right.$ admits a solution $\}$ is finite and for every $t_{0}<t^{*}$ there exists a continuum $\mathcal{C}$ in $\Sigma \equiv\left\{(t, u) \in \mathbb{R} \times C_{0}^{1}(\bar{\Omega}): u\right.$ is a solution of $\left.\left(P_{t}\right)\right\}$ satisfying

1. $\left[t_{0}, t^{*}\right] \subset \operatorname{Proj}_{\mathbb{R}} \mathcal{C}$,

2. for every $t \in\left[t_{0}, t^{*}\right), \operatorname{Proj}_{C_{0}^{1}(\bar{\Omega})}\left[\mathcal{C} \cap\left(\{t\} \times C_{0}^{1}(\bar{\Omega})\right)\right]$ contains two distinct solutions of $\left(P_{t}\right)$.

Remark 2.7. Roughly speaking, the continuum $\mathcal{C}$ of solutions in $\mathbb{R} \times C_{0}^{1}(\bar{\Omega})$ emanates from $\left\{t_{0}\right\} \times C_{0}^{1}(\bar{\Omega})$, reaches $\left\{t^{*}\right\} \times C_{0}^{1}(\bar{\Omega})$ and then it turns left to meet a different solution in $\left\{t_{0}\right\} \times C_{0}^{1}(\bar{\Omega})$ (つ-shaped continuum). As a consequence,

1. $\left(P_{t}\right)$ has at least two solutions for $t<t^{*}$,

2. $\left(P_{t}\right)$ has at least one solution for $t \leq t^{*}$,

3. $\left(P_{t}\right)$ has no solution for $t>t^{*}$.

Proof of Theorem 2.6. Set $S \equiv\left\{t \in \mathbb{R}:\left(P_{t}\right)\right.$ admits a solution $\}$. First we show that $S$ is not empty. This relies upon two facts [12]:

- $\left(P_{t}\right)$ has a supersolution for some $t \in \mathbb{R}$,

- given a supersolution, $\bar{u}$, of $\left(P_{t}\right)$ for some $t \in \mathbb{R}$, there exists a subsolution $\underline{u}$ of it such that $\underline{u}<\bar{u}$ in $\Omega$.

Indeed, by means of the sub-super-solution method, this implies that $S$ is a closed interval unbounded from below. Moreover, the usual trick of multiplying by one positive eigenfunction associated to $\lambda_{1}$ leads to the nonexistence of a solution for $t \gg 0$ large enough and thus $S$ is bounded from above. This means that the supremum of the closed interval $S$ is attained. Define

$$
t^{*} \equiv \sup S=\max S .
$$

We now prove the existence of a continuum of solutions. First we observe that, from Lemma 2.3 jointly with some regularity results, if $t_{0}<t^{*}<t_{1}$, there exists $R>0$ such that $\|u\|_{C^{1}}<R$ for each solution $u$ of $\left(P_{t}\right)$ with $t \in\left[t_{0}, t_{1}\right]$. Denote by $\Phi_{t}$ the map $\Phi_{t}(u) \equiv u-(-\Delta)^{-1}(f(x, u)+t \varphi)$. Using the homotopy invariance of Leray-Schauder degree and the fact that problem $\left(P_{t_{1}}\right)$ has no solution, we get

$$
\operatorname{deg}\left(\Phi_{t}, B_{R}(0), 0\right)=\operatorname{deg}\left(\Phi_{t_{1}}, B_{R}(0), 0\right)=0, \quad \forall t \in\left[t_{0}, t_{1}\right]
$$

where $B_{R}(0)$ denotes the open ball in $C_{0}^{1}(\bar{\Omega})$ of radius $R$ centered at zero.

Let $u^{*}$ be a solution of $\left(P_{t^{*}}\right)$. Observe that $u^{*}$ is a supersolution of $\left(P_{t}\right)$ for every $t \in$ $\left[t_{0}, t^{*}\right)$ and it is not a solution. Moreover, as mentioned above, there exists a subsolution $u_{t_{0}}<u^{*}$ of $\left(P_{t_{0}}\right)$ which is not a solution. Clearly $u_{t_{0}}$ is also a subsolution and no solution for $\left(P_{t}\right)$ if $t \in\left[t_{0}, t^{*}\right)$. Consider the set

$$
U_{1}=\left\{u \in C_{0}^{1}(\bar{\Omega}): u_{t_{0}}<u<u^{*} \text { in } \Omega, \frac{\partial u^{*}}{\partial n}<\frac{\partial u}{\partial n}<\frac{\partial u_{t_{0}}}{\partial n} \text { on } \partial \Omega\right\} \cap B_{R}(0) .
$$

The strong maximum principle implies the nonexistence of solutions of $\left(P_{t}\right)$ on $\partial U_{1}$ if $t<t^{*}$ (see [15]). Hence, the degree of $\Phi_{t}$ is well defined in $U_{1}$. In addition, by using the results in [11],

$$
\operatorname{deg}\left(\Phi_{t}, U_{1}, 0\right)=1, \quad \forall t \in\left[t_{0}, t^{*}\right)
$$


Applying Lemma 2.5 with $E=C_{0}^{1}(\bar{\Omega}),[a, b]=\left[t_{0}, t_{1}\right]$ and $U=B_{R}(0)$, we deduce the existence of a continuum $\mathcal{C}$ in $\Sigma$ such that

$$
\mathcal{C} \cap\left(\left\{t_{0}\right\} \times U_{1}\right) \neq \emptyset, \quad \mathcal{C} \cap\left(\left\{t_{0}\right\} \times\left[B_{R}(0) \backslash \bar{U}_{1}\right]\right) \neq \emptyset .
$$

In particular, the continuum $\mathcal{C}$ crosses $\{t\} \times \partial U_{1}$ for some $t \in\left(t_{0}, t^{*}\right]$. It has been observed that this is possible if and only if $t=t^{*}$. This concludes the proof.

\section{Ambrosetti-Rabinowitz via Ambrosetti-Prodi}

Consider a $C^{1}$-function $f: \bar{\Omega} \times \mathbb{R}^{+} \rightarrow \mathbb{R}^{+}$such that $f(x, 0)=0(x \in \Omega)$, satisfying (6) and

$$
\limsup _{s \rightarrow 0^{+}} \frac{f(x, s)}{s} \leq \gamma<\lambda_{1} \quad \text { uniformly } x \in \Omega .
$$

We are interested in the existence of positive solutions for the b.v.p.

$$
\begin{aligned}
-\Delta u & =f(x, u), & & x \in \Omega, \\
u & =0, & & x \in \partial \Omega .
\end{aligned}
$$

As usual, we can assume that $f$ is extended to all of $\Omega \times \mathbb{R}$ by setting $f(x, s)=0$ for $s<0$. Notice that by the maximum principle, every nontrivial solution of (10) with the extended nonlinearity is positive.

Since $f$ satisfies conditions (3), (5) and (6), problem (10) fits into the "superlinear" Ambrosetti-Prodi framework (problem $\left(P_{t}\right)$ with $t=0$ and any $\varphi$ ). As an easy application of Theorem 2.6, we deduce

Theorem 3.1. Let $f \in C^{1}\left(\bar{\Omega} \times \mathbb{R}^{+}\right)$satisfy (6) and (9). Then problem (10) has at least one positive solution.

Proof. To apply the framework of the previous sections we embed (10) into the oneparameter family

$$
\begin{aligned}
-\Delta u & =f(x, u)+t \varphi_{1}, & & x \in \Omega, \\
u & =0, & & x \in \partial \Omega .
\end{aligned}
$$

Observe that this problem is $\left(P_{t}\right)$ with $\varphi=\varphi_{1}$. From Theorem 2.6, let $t^{*}$ denote the supremum of all $t \in \mathbb{R}$ such that $\left(Q_{t}\right)$ has a solution. The proof is concluded if we prove that $t^{*}>0$. Indeed, if this is done, that theorem (see also item 1 of Remark 2.7 with $t=0)$ shows the existence of at least two solutions of $\left(Q_{0}\right)$, or equivalently, the existence of two nonnegative solutions of (10). Taking into account that zero is a solution of this problem, we deduce that there exists at least one positive solution of (10).

In order to prove that $t^{*}>0$, we use the sub-super-solution method. First, we note that $u=0$ is a subsolution of $\left(Q_{t}\right)$ for every $t>0$. Thus, we only have to find a positive supersolution of $\left(Q_{t}\right)$ for some $t>0$. This is easily deduced as follows. From (9), we take $\delta$ small enough such that $f\left(x, \delta \varphi_{1}\right) / \delta \varphi_{1} \leq \lambda_{1}-\delta$ for every $x \in \Omega$. Then

$$
-\Delta\left(\delta \varphi_{1}\right)=\lambda_{1} \delta \varphi_{1} \geq f\left(x, \delta \varphi_{1}\right)+\delta^{2} \varphi_{1} .
$$

This means that $\delta \varphi_{1}$ is a supersolution of $\left(Q_{t}\right)$ with $t=\delta^{2}>0$, concluding the proof. 
Acknowledgments. Both authors are partially supported by D.G.E.S. Ministerio de Educación y Ciencia (Spain) n. BFM2003-03772.

\section{References}

[1] Amann, H., Hess, P.: A multiplicity result for a class of elliptic boundary value problems. Proc. Roy. Soc. Edinburgh Sect. A 84, 145-151 (1979) Zbl 0416.35029 MR 0549877

[2] Ambrosetti, A., Prodi, G.: A Primer on Nonlinear Analysis. Cambridge Stud. Adv. Math. 34, Cambridge Univ. Press, Cambridge (1995) Zbl 0818.47059 MR 1336591

[3] Ambrosetti, A., Prodi, G.: On the inversion of some differentiable mappings with singularities between Banach spaces. Ann. Mat. Pura Appl. 93, 231-246 (1972) Zbl 0288.35020 MR 0320844

[4] Ambrosetti, A., Rabinowitz, P. H.: Dual variational methods in critical point theory and applications. J. Funct. Anal. 14, 349-381 (1973) Zbl 0273.49063 MR 0370183

[5] Arcoya, D., Carmona, J.: Quasilinear elliptic problems interacting with its asymptotic spectrum. Nonlinear Anal. 52, 1591-1616 (2003) Zbl 1013.35022 MR 1951509

[6] Arcoya, D., Ruiz, D.: The Ambrosetti-Prodi problem for the $p$-Laplace operator. Comm. Partial Differential Equations, to appear

[7] Berestycki, H.: Le nombre de solutions de certains problèmes semi-linéaires. J. Funct. Anal. 40, 1-29 (1981) Zbl 0452.35038 MR 0607588

[8] Berger, M. S., Podolak, E.: On the solutions of a nonlinear Dirichlet problem. Indiana Univ. Math. J. 24, 837-846 (1975) Zbl 0329.35026 MR 0377274

[9] Brézis, H., Nirenberg, L.: $H^{1}$ versus $C^{1}$ local minimizers. C. R. Acad. Sci. Paris Sér. I Math. 317, 465-472 (1993) Zbl 0803.35029 MR 1239032

[10] Chang, K. C.: Variational methods and sub- and super-solutions. Sci. Sinica Ser. A 26, 12561265 (1983) Zbl 0544.35045 MR 0745797

[11] de Figueiredo, D. G.: Lectures on boundary value problems of the Ambrosetti-Prodi type. Atas do $12^{0}$ Seminário Brasileiro de Análise, São Paulo, Brazil (1980)

[12] de Figueiredo, D. G.: Lectures on the Ekeland Variational Principle with Applications and Detours. Tata I.F.R. Lectures on Mathematics and Physics 81, Springer (1989) Zbl 0688.49011 MR 1019559

[13] de Figueiredo, D. G., Solimini, S.: A variational approach to superlinear elliptic problems. Comm. Partial Differential Equations 9, 699-717 (1984) Zbl 0552.35030 MR 0745022

[14] Fučik, S.: Remarks on a result by A. Ambrosetti and G. Prodi. Boll. Un. Mat. Ital. (4) 11, 259-267 (1975) Zbl 0303.35037 MR 0382849

[15] Gámez, J. L.: Sub- and super-solutions in bifurcation problems. Nonlinear Anal. 28, 625-632 (1997) Zbl 0865.35015 MR 1420381

[16] Gidas, B., Spruck, J.: A priori bounds for positive solutions of nonlinear elliptic equations. Comm. Partial Differential Equations 6, 883-901 (1981) Zbl 0462.35041 MR 0619749

[17] Lefton, L. E., Shapiro, V. L.: Quasilinear ellipticity and jumping nonlinearities. Rocky Mountain J. Math. 22, 1385-1403 (1992) Zbl 0786.35056 MR 1201100

[18] Mawhin, J.: The periodic Ambrosetti-Prodi problem for nonlinear perturbations of the $p$ Laplacian. This volume, 375-388

[19] Stampacchia, G.: Le problème de Dirichlet pour les équations elliptiques du second ordre à coefficients discontinus. Ann. Inst. Fourier (Grenoble) 15, 189-258 (1965) Zbl 0151.15401 MR 0192177 